\title{
ABSTRACT ERGODIC THEOREMS AND WEAK ALMOST PERIODIC FUNCTIONS $\left({ }^{1}\right)$
}

\author{
BY \\ W. F. EBERLEIN
}

Although the individual ergodic theorem of G. D. Birkhoff is sharper than the mean ergodic theorem of J. von Neumann, it was soon evident that the measure-theoretic formulation obscured the greater generality of the latter result. Thus various authors-notably F. Riesz [28] and Yosida and Kakutani [33] -extended the mean ergodic theorem to an abstract theorem asserting the convergence to a fix point of the means $T_{n} x=(n+1)^{-1} \sum_{0}^{n} T^{i} x$, where $T$ is a linear transformation of a Banach space $E$ into itself. Alaoglu and Birkhoff [1] then replaced the iterates $\left(T^{j}\right)$ by a semi-group $G$ of linear transformations and showed that convergence of certain general means of transforms of an element $x$ of $E$ is equivalent to the existence and uniqueness of a fix point $y$ in the closed convex hull of the orbit of $x$ under $G$. The persistence of the customary countability and uniform boundedness restrictions on $G$ in their work, however, severely limits the generality. A fresh abstraction is thus required, not only to subsume present results in a sharper and more transparent form, but to extend the domain of the ergodicity phenomena.

In Part I we study a semi-group $G$ of linear transformations operating on a space $\varepsilon, G$ ordinarily being restricted by an "ergodicity" condition of the weakest type. We derive criteria for the validity of a mean ergodic theorem in an arbitrary locally-convex linear topological space $\varepsilon$. Specializing $\varepsilon$ to a Banach space $E$, we obtain not only standard theorems as obvious corollaries of the general theory, but significantly new results. For example, we obtain a mean ergodic theorem for an arbitrary bounded Abelian semi-group $G$ on the one hand, and Fejér's theorem as an ergodic theorem for an unbounded semigroup on the other. The role played by weak compactness in $E$ and (weak) quasi-compactness of the operators $T$ of $G$ is clarified, as well as the relation of ergodic theory to the mean value problem for generalized almost periodic functions, the relation being particularly simple in case the underlying group is Abelian.

In Part II we consider a locally-compact Abelian group $G$ acting as translations on the Banach algebra $C(G)$ of bounded continuous complex-valued

Presented to the Society, April 16, 1948, and September 9, 1948; received by the editors August 11, 1948.

(1) Prepared under the sponsorship of the Office of Naval Research (Contract N7onr-379 T.O. II, NRO43-051). A summary of most of Part I appeared in [14]. Numbers in brackets refer to the bibliography at the end of the paper. 
functions on $G$. Calling $x(t)$ in $C(G)$ weakly almost periodic (w.a.p.) if the set of translates $x(t+s)(s \in G)$ is (conditionally) weakly compact, we study the class $\mathfrak{W}$ of w.a.p. functions. The principal results are: (1) the existence of the mean value as an ergodic theorem; (2) the closure of $\mathfrak{B}$ under multiplication, in contrast to other classes of generalized a.p. functions; (3) the fact that $\mathfrak{W}$ contains all the bounded functions pertinent to Fourier analysis on Abelian groups-for example, almost periodic functions, positive definite functions, and continuous functions vanishing at infinity; (4) the uniform continuity of w.a.p. functions; (5) the almost periodicity of the convolution of two w.a.p. functions; (6) the Parseval equation for Fourier expansions in the characters of $G$; (7) if $G=R_{1}$, that a w.a.p. function is in the Weyl class $\mathrm{W}^{p}$ for every $p \geqq 1$.

The equivalence of the properties of compactness (=bicompactness), countable compactness, and sequential compactness for (closed) sets in the weak topology of a Banach space [13] is vital to the theory, particularly since we impose no countability restrictions on $G$.

Terminology. Linearity of an operator denotes continuity and total definition, as well as linearity in the algebraic sense. A set of operators forms a semigroup if it is closed under operator multiplication.

\section{Part I. Abstract ERgodic theorems}

1. Weak topology. We summarize some properties of the weak topology of linear topological spaces (1.t.s.) that are required later. (For the definition and elementary properties of 1.t.s. see, for example, Hyers [20].)

Given an 1.t.s. $\varepsilon$ denote by $\varepsilon^{*}$ the set of all linear functionals defined on $\varepsilon$. In the only case we consider $\varepsilon$ is locally convex, whence there exist "sufficiently many" linear functionals - that is, given $x \neq 0$ in $\varepsilon$ there exists an $f$ in $\varepsilon^{*}$ such that $f(x) \neq 0$. The elements of $\varepsilon$ then form a new l.t.s. (weak topology of $\varepsilon$ ) under the system of neighborhoods $N\left(x ; f_{1}, \cdots, f_{n}, \epsilon\right)=\left[y|| f_{i}(x-y) \mid\right.$ $<\epsilon, i=1, \cdots, n]$, where $\epsilon>0$ is arbitrary, and $\left(f_{1}, \cdots, f_{n}\right)$ is an arbitrary finite set of elements of $\varepsilon^{*}$. It follows trivially that the weak closure of a set $M$ in $\varepsilon$ implies the strong closure. When $M$ is convex, the converse is valid and provides the fundamental connection between the two topologies:

THEOREM 1.1 (MAZUR-Bourgin [6]). In a locally convex l.t.s. a closed convex set is weakly closed.

The interest of the weak topology is largely due to the vital role played by weak compactness in the theory of infinite-dimensional linear spaces. When $\varepsilon$ is a Banach space the weak compactness theory is almost metric in its good behavior, and we shall be interested primarily in this case. The fundamental results we need are: (1) that the closed convex hull of a conditionally weakly compact set is weakly compact [26]; (2) that the various standard notions of weak compactness are equivalent [13]. For our purposes these facts 
are conveniently summarized in

TheOREM 1.2. Let $E$ denote a Banach space, $M$ a subset of $E$, and $\Sigma$ the closed convex hull of $M$. In the weak (as well as in the metric) topology of $E$ the following conditions are equivalent:

(1) $\Sigma$ is compact (=bicompact);

(2) $M$ is conditionally sequentially compact-that is, every sequence $\left(x_{n}\right)$ in $M$ contains a subsequence $\left(x_{n_{i}}\right)$ such that $\lim _{n_{i}} x_{n_{i}}=x$ for some $x$ in $E$;

(3) $M$ is conditionally countably compact-that is, every infinite subset of $M$ possesses at least one limit point $x$ in $E$.

In the weak topology of a Banach space, therefore, we need distinguish only conditional compactness and compactness, depending on the closure of the set involved, and we may employ sequential compactness notions freely. The crucial role played by sequential weak convergence is apparent in the case $E=C(\Omega)=$ all continuous (real- or complex-valued) functions $x(t)$ on a compact Hausdorff space $\Omega$ with $\|x\|=\sup _{t \in \Omega}|x(t)|$ :

THEOREM 1.3. In $C(\Omega), x(t)=\lim _{n} x_{n}(t)$ weakly if and only if (1) $\left\|x_{n}\right\|$ is bounded; (2) $\lim _{n} x_{n}(t)=x(t)$ for every $t \in \Omega$.

Since the general linear functional $f(x)$ on $C(\Omega)$ has the form of a Radon integral

$$
f(x)=\int_{\Omega} x(t) d \mu(t),
$$

where $\mu$ is a countably additive regular Borel set function of finite total variation on $\Omega$ [22], the nontrivial half of the above theorem essentially amounts to the Lebesgue convergence theorem. Moreover, not only this proof but the theorem itself breaks down [29] on replacing the sequence $x_{n}$ by a directed set $x_{\alpha}$.

We note also that the above remarks remain valid for a locally compact Hausdorff space $\Omega$ if $E=C_{0}(\Omega)$ now denotes the continuous functions vanishing at infinity. $(x(t)$ vanishes at infinity if given $\epsilon>0$ there exists a compact set $\Gamma \subset \Omega$ such that $|x(t)| \leqq \epsilon$ for $t \in \Omega-\Gamma$.)

2. Ergodic semi-groups. Consider now a locally convex 1.t.s. $\mathcal{E}$ and a semigroup $G$ of linear transformations of $\varepsilon$ into itself. We denote by $G^{*}$ $=\left[\sum a_{j} T_{j} \mid a_{j} \geqq 0, \sum a_{i}=1, T_{j} \in G\right]$ the family of transformations $T^{*}$ consisting of all finite convex combinations of the elements $T$ of $G$; by $O(x)$ $=\left[T^{*} x \mid T^{*} \in G^{*}\right]$ the orbit of $x$ under $G^{*}$, and by $\bar{O}(x)$ the closure of $O(x)$ in $\varepsilon$. $G^{*}$ is clearly a semi-group of operators, and $O(x)$ and $\bar{O}(x)$ are convex sets invariant under $G^{*}$. For convenience we shall always assume that $G$ contains the identity $I$.

The semi-groups $G$ of chief interest to us are those with the property we denote as ergodicity. 
DEFINITION 2.1. G is an ergodic semi-group if it possesses at least one system of almost invariant integrals. By such a system we mean a family of transformations $\left(T_{\alpha}\right)$, indexed by the directed set $(\alpha)$, with the following properties:

I. $T_{\alpha}$ is a linear transformation of $\varepsilon$ into itself for every $\alpha$.

II. For every $x$ and all $\alpha T_{\alpha} x \in \bar{O}(x)$.

III. The transformations $T_{\alpha}$ are equi-continuous.

IV. For every $x$ in $\varepsilon$ and all $T$ in $G$ :

(a) $\lim _{\alpha}\left(T T_{\alpha} x-T_{\alpha} x\right)=0$;

(b) $\lim _{\alpha}\left(T_{\alpha} T x-T_{\alpha} x\right)=0$.

We shall see later that the problem of the existence or construction of a system of almost invariant integrals for a given semi-group $G$ is a difficult one. In many cases of interest, however, the system appears naturally, and a complete solution is possible in the important bounded Abelian case ( $\$ 5$, Example 3). Moreover, it turns out that any two systems of almost invariant integrals are equivalent for our purposes, whence ergodicity is a property of the semi-group $G$ rather than of the particular system exhibited.

The following remarks are easy consequences of the above definition: It is clear that IV remains valid if $T$ is replaced by any element $T^{*}$ of $G^{*}$. III follows automatically from II when the $T$ in $G$ are assumed equi-continuous. (As we shall see later, however, this restriction on $G$ is unduly stringent.) When $\varepsilon$ is a Banach space $E$, III and IV take the equivalent forms

III': There exists $M>0$ such that $\left\|T_{\alpha}\right\| \leqq M$ for all $\alpha$;

IV': For all $T$ in $G, \lim \left(T T_{\alpha}-T_{\alpha}\right)=\lim \left(T_{\alpha} T-T_{\alpha}\right)=0$ "strongly" in $B(E)$, where $B(E)$ is the Banach algebra of linear transformations of $E$ into itself. We sometimes consider the case where the limits IV' hold in the "uniform" (=norm) topology of $B(E)$ as well as in the "strong"; and in either case it is evident that the content of $I V^{\prime}$ is not altered on replacing $T$ by an element $U$ of $\bar{G}^{*}$, where the semi-group $\bar{G}^{*}$ is the norm closure of $G^{*}$ in $B(E)$. Moreover, it is trivial that $U x \in \bar{O}(x)$ for every $U \in \bar{G}^{*}$.

3. The mean ergodic theorem. Our definition of an ergodic element of $\varepsilon$ is inspired by the following key theorem, valid in any locally convex 1.t.s.

THEOREM 3.1. If $G$ is ergodic, $x$ an element of $\varepsilon$, and $\left(T_{\alpha}\right)$ any system of element invariant integrals, then the following conditions on an element $y$ in $\varepsilon$ are equivalent:

(1) $y \in \bar{O}(x)$, and $T y=y$ for all $T \in G$;

(2) $y=\lim _{\alpha} T_{\alpha} x$

(3) $y=\lim _{\alpha} T_{\alpha} x$ weakly;

(4) $y$ is a weak cluster point $\left({ }^{2}\right)$ of $\left(T_{\alpha} x\right)$.

DEFINITION 3.1. If $G$ is ergodic, $x$ is ergodic with (unique) limit fix point $y$

(2) $y$ is a cluster point of the directed set $\left(x_{\alpha}\right)$ in a $T$-space if for each $\beta$ and each nbd. $U$ of $y$ there exists an $\alpha>\beta$ such that $z_{\alpha} \in U$. The compactness of the closure of $\left(x_{\alpha}\right)$ implies the existence of at least one cluster point $y$ (cf. Tukey [31]). 
if and only if there exists a $y=T_{\infty} x$ satisfying any of the conditions (1) to (4) above.

The justification for this definition rests on the following observations: Since condition (1) is independent of the particular system $\left(T_{\alpha}\right)$, the validity of (2), (3), or (4) for any system $\left(T_{\alpha}\right)$ implies-by way of (1) - the validity for any other system $\left(T_{\alpha}^{\prime}\right)$. Hence the property of being an ergodic element is independent of the particular system of almost-invariant integrals employed, provided at least one system exists. Moreover, (2) implies that the limiting fix point $y$, if it exists, is necessarily unique.

Turning now to the proof of Theorem 3.1, we note that the implications $(2) \rightarrow(3) \rightarrow(4)$ are trivial. We complete the proof by establishing $(1) \rightarrow(2)$ and $(4) \rightarrow(1)$.

$(1) \rightarrow(2)$. Given any nbd. $N(0) \subset \varepsilon$ we must exhibit a $\beta$ such that $\alpha>\beta$ implies $y-T_{\alpha} x \in N(0)$. Choose a nbd. $V(0)$ such that $V+V \subset N$, and a nbd. $W(0)$ such that $T_{\alpha}(W) \subset V$ for all $\alpha$ (III). Since $y$ is in $\bar{O}(x)$ there exists, by definition, $T^{*} \in G^{*}$ such that $y-T^{*} x \in W$. Now choose $\beta$ such that $\left(T_{\alpha} T^{*} x-T_{\alpha} x\right) \in V$ if $\alpha>\beta(\mathrm{IVb})$. Recalling that II implies $T_{\alpha} y=y$ for a fix point $y$, we can now write

$y-T_{\alpha} x=T_{\alpha}\left(y-T^{*} x\right)+\left(T_{\alpha} T^{*} x-T_{\alpha} x\right) \in T_{\alpha}(W)+V \subset V+V \subset N(0)$

for $\alpha>\beta$.

$(4) \rightarrow(1)$. Since $T_{\alpha} x \in \bar{O}(x)$ for all $\alpha$ (II), the $y$ in (4) is a weak limit point of $\bar{O}(x)$, whence $y \in \bar{O}(x)$ by the Mazur theorem. To prove that $T y=y$ for all $T \in G$ note that, since there are sufficiently many linear functionals, it is sufficient to show that $f(T y-y)=0$ for all $T$ in $G$ and all $f$ in $\varepsilon^{*}$. Given then arbitrary $\epsilon>0, T \in G$, and $f \in \mathcal{E}^{*}$, we note first that setting $g(x)=f(T x)$ defines an element $g \in \mathcal{E}^{*}$. Since strong convergence implies weak convergence, IV (a) implies the existence of an index $\beta$ such that

$$
\left|f\left(T_{\alpha} x-T T_{\alpha} x\right)\right|<\epsilon / 3 \quad(\alpha>\beta) .
$$

Since $y$ is a weak cluster point of $T_{\alpha} x$ there exists some $\alpha>\beta$ such that $y-T_{\alpha} x \in N(0 ; f, g, \epsilon / 3)$ - that is,

$$
\begin{gathered}
\left|f\left(y-T_{\alpha} x\right)\right|<\epsilon / 3, \\
\left|f\left(T T_{\alpha} x-T y\right)\right|=\left|g\left(T_{\alpha} x-y\right)\right|<\epsilon / 3 .
\end{gathered}
$$

Adding these inequalities we obtain $|f(y-T y)|<\epsilon$. Since $\epsilon>0, T \in G, f \in \varepsilon^{*}$ were arbitrary, $T y=y$ for all $T \in G$.

REMARK. We note that IV(a) was required only to establish (4) $\rightarrow(1)$, IV (b) only in proving (1) $\rightarrow(2)$, and in either case only the validity at the point $x$ was needed.

4. The ergodic and almost periodic subspaces. Before proceeding to the applications of our mean ergodic theorem we shall examine the space of 
ergodic elements and its important subspaces. We assume henceforth that $\varepsilon$ is a Banach space $E$.

THEOREM 4.1. If $G$ is ergodic, the ergodic elements of $E$ constitute a closed invariant linear subspace $\Gamma$. The transformation $T_{\infty} x=y=\lim _{\alpha} T_{\alpha} x$ is a linear transformation of $\Gamma$ into itself, and $\left\|T_{\infty}\right\|_{\Gamma} \leqq M$. Moreover, on $\Gamma$ we have $T_{\infty}=T_{\infty}^{2}$ $=T_{\infty} U=U T_{\infty}$ if $U \in \bar{G}^{*}$ or $U=$ some $T_{\alpha}$.

Proof. Theorem 3.1 (2) clearly implies that $\Gamma$ is linear and $\left\|T_{\infty} x\right\| \leqq M\|x\|$ for all $x \in \Gamma$. If $x \in \Gamma, \operatorname{IV}(\mathrm{b})$ implies $T_{\infty} T x=\lim _{\alpha} T_{\alpha} T x=\lim _{\alpha} T_{\alpha} x=T_{\infty} x$ exists, whence $\Gamma$ is invariant under $G$. The proof that $\Gamma$ is closed involves the equicontinuity condition III': Assuming $\left(x_{n}\right) \subset \Gamma, x=\lim x_{n}, y_{n}=\lim _{\alpha} T_{\alpha} x_{n}$, note that $\left\|y_{n}-y_{m}\right\|=\| T_{\infty}\left(x_{n}-x_{m}\|\leqq M\| x_{n}-x_{m} \|\right.$, whence the sequence is $y_{n}$ fundamental and $y=\lim y_{n}$ exists. That $y=\lim _{\alpha} T_{\alpha} x$ then follows from the inequality

$$
\left\|T_{\alpha} x-y\right\| \leqq\left\|T_{\alpha}\left(x-x_{N}\right)\right\|+\left\|T_{\alpha} x_{N}-y_{N}\right\|+\left\|y_{N}-y\right\|
$$

on choosing $N$ sufficiently large, and then $\alpha$ suitably.

The remaining statements of the theorem can be given the following more general setting, in which the ergodicity of $G$ is not assumed.

LeMma 4.1. If, for every point $x$ of a closed invariant linear subspace $\Gamma$, $\bar{O}(x)$ contains a unique fix point $T_{\infty} x$, and if $U \in B(E)$ has the property $U x$ $\in \bar{O}(x)$ for every $x \in \Gamma$, then $T_{\infty} x=T_{\infty}^{2} x=U T_{\infty} x=T_{\infty} U x$.

To establish these equalities note first that $U T_{\infty} x \in \bar{O}\left(T_{\infty} x\right)=T_{\infty} x$. Hence $U T_{\infty} x=T_{\infty} x$; similarly, $T_{\infty}^{2} x=T_{\infty} x$. To prove $T_{\infty} U x=T_{\infty} x$, note that $U x$ $\in \bar{O}(x) \subset \Gamma, \bar{O}(U x) \subset \bar{O}(x)$, whence the fix point $T_{\infty} U x$ exists, lies in $\bar{O}(x)$, and is necessarily $T_{\infty} x$. The proof of the theorem is then complete.

The structure of the ergodic subspace having been established, we now investigate conditions sufficient to insure that a given element $x$ of $E$ be ergodic. In this connection we shall find (4) of Theorem 3.1 to be the most useful criterion for ergodicity, and weak compactness the most useful condition: For example, the required $y$ exists automatically whenever any of the sets $\bar{O}(x),\left(T_{\alpha} x\right)$, or $[T x \mid T \in G]$ is (conditionally) weakly compact. (Footnote 2 and Theorem 1.2.)

This remark and reference to the theory of almost periodic points of transformation groups suggest consideration of the following subspaces of the ergodic space $\Gamma$.

DEFINITION 4.1. $x \in E$ is (weakly) almost periodic under $G$ if and only if the orbit $[T x \mid T \in G]$ is conditionally (weakly) compact. $\mathfrak{W}$ and $\mathfrak{U}$ denote the set of weak and strong almost periodic points respectively.

The inclusion relations $\mathfrak{U} \subset \mathfrak{B} \subset \Gamma \subset E$ are obvious when $G$ is ergodic. When $G$ is bounded, but not necessarily ergodic, one can establish 
Theorem 4.2. If $\|T\| \leqq M$ for all $T \in G$, the (weak) almost periodic subspace (W) $\mathfrak{U}$ is a closed linear invariant subspace of $E$.

Proof. The linearity and invariance under $G$ of $\mathfrak{U}$ and $\mathfrak{W}$ are obvious. Closure of $\mathfrak{U}$ follows from an obvious application of the diagonal process and the completeness of $E$ in the norm topology. The incompleteness of $E$ in the weak topology, however, necessitates modifications in the proof of closure for $\mathfrak{W}$. Fortunately we can still restrict consideration to sequential compactness notions (Theorems 1.2).

Assuming $x=\lim x_{n},\left(x_{n}\right) \subset \mathfrak{W}$, and given any sequence $\left(T_{l}\right)\left(T_{l} \in G\right)$, we derive via the diagonal process a subsequence $\left(T_{l^{\prime}}\right) \subset\left(T_{l}\right)$ and a set of elements $y_{n}$ such that $\lim _{l^{\prime}} T_{l^{\prime}} x_{n}=y_{n}$ weakly $(n=1,2, \cdots)$. If we could show that $y=\lim _{n} y_{n}$ exists, it would follow readily that $y=\lim _{l^{\prime}} T_{l^{\prime}} x$ weakly, whence $x \in \mathfrak{W}$. But the Hahn-Banach theorem [2] implies that $\left\|y_{m}-y_{n}\right\|$ $=\sup _{\|f\|=1}\left|f\left(y_{m}-y_{n}\right)\right|=\sup _{\|f\|=1} \lim _{l^{\prime}}\left|f\left\{T_{l^{\prime}}\left(x_{m}-x_{n}\right)\right\}\right| \leqq M\left\|x_{m}-x_{n}\right\|$. Hence $\left(y_{n}\right)$ is fundamental, and $y=\lim _{n} y_{n}$ exists.

5. Examples and applications. We now justify our description of Theorem 3.1 as a mean ergodic theorem by deriving from it in elementary fashion essentially all standard mean ergodic theorems. We further obtain important applications to Fourier analysis. The derivation consists of (1) verifying the ergodicity (Definition 2.1) of the particular semi-group $G$ considered, and (2) verifying the ergodicity of the particular element $x$ (Definition 3.1) by producing the required $y$-usually by a weak compactness argument.

The following examples are all of the Abelian type, whence (a) and (b) of IV coalesce. The more difficult noncommutative case will be discussed in $\S 8$.

Example 1. Standard Case. Let $G$ consist of the iterates $\left(T^{n}\right)$ $(n=0,1, \cdots)$ of a linear transformation $T$, and let the $T_{\alpha}$ be the Cesàro means

$$
T_{n}=(n+1)^{-1} \sum_{0}^{n} T^{j}
$$

under the natural ordering. I and II are trivially satisfied, and the validity of IV hinges on the identity

$$
T^{m} T_{n}-T_{n}=(n+1)^{-1}\left(\sum_{n+1}^{n+m} T^{j}-\sum_{0}^{m-1} T^{j}\right) \quad(m \geqq 1) .
$$

The usual condition that $\left\|T^{n}\right\| \leqq M$ for all $n$ then insures the validity of III, and of IV in the uniform sense. However, as pointed out in examples by Dunford [10] and Hille [19], uniform boundedness of the $T^{n}$ is too restrictive $\left(^{3}\right)$ : all one needs is a bound on the $\left\|T_{n}\right\|$ and $\lim _{n} T^{n} / n=0$ in an appropriate sense.

( $\left.{ }^{3}\right)$ If $E=$ Hilbert space and $\left\|T^{n}\right\| \leqq M(n=0, \pm 1, \pm 2, \cdots), T$ is effectively no more yen. eral than a unitary transformation (cf. de Sz. Nagy [30]). 
Theorem 3.1 then specializes to

Theorem 5.1. Let $T \in B(E)$ be such that $\left\|T_{n}\right\|$ is bounded and $\lim _{n} T^{n} x / n=0$ for every $x$. If the set $\left(T_{n} x\right)$ is conditionally weakly compact, then $y=\lim _{n} T_{n} x$ exists and $T y=y$.

For the sake of completeness we note some special cases of interest: When $E$ is reflexive every (metrically) bounded set is conditionally weakly compact [13], whence every $x$ in $E$ is ergodic. This remark covers Hilbert space and, more generally, the spaces $L^{p}$ and $l^{p}(p>1)$. In the spaces $L$ and $l$ Riesz [28], Birkhoff [1], Kakutani [33] and others observed that a lattice-theoretically bounded set of functions-that is, $[x \mid a(t) \leqq x(t) \leqq b(t)$ identically, $a, b \in L$ or $l]$-is conditionally weakly compact. In a Lebesgue space $L(S)$ a general criterion for weak compactness (Riesz [28], Dunford and Pettis [12]) yields the conditional weak compactness of $T_{n} x$ if $T$ is of the classical form $T x(t)$ $=x(\phi t)$, where $\phi$ is a one-to-one measure-preserving transformation of $S$ into itself $\left({ }^{4}\right)$.

The next example illustrates the fact that our definition of ergodicity subsumes continuous as well as discrete means.

Example 2. Almost periodic functions. Let $E$ denote the Banach space of complex-valued bounded uniformly continuous functions $x(t)(-\infty<t<\infty)$ with $\|x\|=\sup _{t}|x(t)|$. Let $G$ be the group of translations $U_{a} x(t)=x(t+a)$, whose only fix points are the constant functions. One may take as the system $\left(T_{\alpha}\right)$ the Bohr means

$$
T_{\alpha} x(t)=\frac{1}{\alpha} \int_{0}^{\alpha} x(t+s) d s
$$

under the natural ordering. It is obvious that $T_{\alpha}$ is linear (I) and that $\left\|T_{\alpha}\right\|=1\left(\mathrm{III}^{\prime}\right)$. That IV holds in the uniform sense follows from the inequality

$$
\left\|U_{a} T_{\alpha} x-T_{\alpha} x\right\|=\frac{1}{\alpha}\left\|\int_{a}^{\alpha+a} x(t+s) d s-\int_{0}^{a} x(t+s) d s\right\| \leqq 2|a| \cdot\|x\| / \alpha .
$$

The proof of II requires uniform continuity to assure the uniform (in $t$ ) approximation of $T_{\alpha} x(t)$ by Riemann sums.

Since an almost periodic function is uniformly continuous, and the set of translates $x(t+a)(-\infty<a<\infty)$ is conditionally compact by definition, the ergodic theorem now yields the existence of the mean value:

TheOREM 5.2(5). If $x(t)(-\infty<t<\infty)$ is almost periodic, the limit $T_{\infty} x$

(4) Dunford and Miller [11] show that the conditions on $\phi$ can be weakened if $m(S)<\infty$ and derive the individual ergodic theorem from the mean ergodic theorem.

(5) It is readily verified that our methods will yield Kawada's [22] extension of this formula to a locally compact Abelian connected group. 
$=M=\lim _{\alpha \rightarrow \infty}(1 / \alpha) \int_{0}^{\alpha} x(t+s) d s=\lim _{\alpha \rightarrow \infty}(1 / \alpha) \int_{z}^{\alpha+t} x(s) d s$ exists uniformly in $t$ and is a constant.

The fact that this conclusion actually requires only the weak conditional compactness of the translates of $x(t)$ provides the motivation for the study (in Part II) of "weak" almost periodic functions defined on locally compact Abelian groups. That the corresponding group of translations in this general case is ergodic is implicit in our next example.

ExAmple 3. Bounded Abelian semi-group. Consider an arbitrary semigroup $G$ that is Abelian and bounded-that is, $\|T\| \leqq M$ for all $T$ in $G$. This hypothesis clearly includes Example 2 and the bounded case of Example 1. We now exhibit a system of almost invariant integrals for $G$.

Order the elements of $G^{*}$ as follows: $U \supset V$ if and only if there exists $W$ in $G^{*}$ such that $U=W V$. That this ordering has the composition property follows from the fact that $U V=V U$ is a common successor of $U$ and $V$. Regarding each element of $G^{*}$ as its own index, we find that the elements of $G^{*}$ under this ordering form a (uniform) system of almost-invariant integrals: For the conditions I, II, and III are trivially satisfied. To establish IV we must show that given $T$ in $G$ and $\epsilon>0$ there exists $U$ in $G^{*}$ such that $T^{*} \supset U$ implies $\left\|T T^{*}-T^{*}\right\|<\epsilon$. Simply take $U$ as the $T_{N}$ of Example $1, N$ being chosen so large that $\left\|T_{N} T-T_{N}\right\|<\epsilon / M$. If $T^{*}=V T_{N}$ is any successor of $T_{N}$, then

$$
\left\|T T^{*}-T^{*}\right\|=\left\|T^{*} T-T^{*}\right\|=\left\|V\left(T_{N} T-T_{N}\right)\right\| \leqq M\left\|T_{N} T-T_{N}\right\|<\epsilon .
$$

We have therefore established

LEMma 5.1. If $G$ is bounded and Abelian, then $G$ is ergodic.

For purposes of reference we now summarize the main features of the bounded Abelian case:

THEOREM 5.3. If $G$ is bounded and Abelian, $x$ is ergodic if and only if there exists a fix point $y$ in $\bar{O}(x) . y$, if it exists, is unique. The conditional weak compactness of $\bar{O}(x)$ or $[T x \mid T \in G]$ implies the existence of a fix point $y$ in $\bar{O}(x)\left(^{6}\right)$.

REMARK. When $E=L_{1}(-\infty, \infty)$ and $G$ is the group of translations $x(t) \rightarrow x(t+a)$, the situation is degenerate: (1) The space of fix points and the almost periodic subspaces $\mathfrak{U}$ and $\mathfrak{W}$ reduce to the null function; (2) the ergodic subspace consists [1] of the functions $x(t)$ such that $\int_{-\infty}^{\infty} x(t) d t=0$. (That the null function is the only function with a conditionally weakly compact set of translates is an easy consequence of that part of the weak compactness criterion [12] peculiar to a $\sigma$-finite measure space.)

$\left(^{6}\right)$ The existence of a fix point in a weakly compact $\delta(x)$ in the Abelian case also follows from a fix point theorem of Markoff [21]. The special case of our theorem in which $E$ is reflexive is due to Day [9]. 
ExAmple 4. Summability of Fourier series. The significance of requiring a bound on the $T_{\alpha}$ (III') rather than on all the $T$ in $G$ will now be clarified. This apparently technical generalization is actually the heart of the summability theory of Fourier series. In fact, we shall exhibit Fejér's theorem as an ergodic theorem for an unbounded semi-group.

Let $C$ denote the space of continuous real-valued functions of period $2 \pi$ with $\|x\|=\sup _{t}|x(t)|$. Denote by $S_{n}$ the transformations $S_{n} x=s_{n}(t)$, where $s_{n}(t)$ is the $n$th partial sum of the Fourier trigonometrical series corresponding to $x$. Let $G$ consist of the identity $I$ and the transformations $U_{n}=I-S_{n}$. The transformations $S_{n}, U_{n}$ are clearly linear; and the identity $U_{n} U_{m}=U_{v}$, where $v=\max (m, n)$, implies that $G$ is an Abelian semi-group. That the only fix point of $G$ is the null function follows from the completeness of the trigonometrical system.

The semi-group $G$ is unbounded. Although the $U_{n}$ themselves trivially satisfy conditions I, II, and IV of a system of almost-invariant integrals for $G$, the $\left\|U_{n}\right\|$ are unbounded. In fact, the Dirichlet representation

$$
s_{n}(t)=\frac{1}{\pi} \int_{-\pi}^{\pi} x(t+u) D_{n}(u) d u
$$

implies that $\left\|S_{n}\right\|=L_{n}=\left(4 / \pi^{2}\right) \log n+O(1)$, where

$$
L_{n}=\frac{1}{\pi} \int_{-\pi}^{\pi}\left|D_{n}(u)\right| d u=\frac{2}{\pi} \int_{0}^{\pi} \frac{|\sin (n+1 / 2) u|}{2 \sin (u / 2)} d u
$$

are the Lebesgue constants $[34$, p. 172].

However, the $(C, 1)$ means $T_{n}=(n+1)^{-1} \sum_{0}^{n} U_{j}$ form a (uniform) system of almost invariant integrals: I and II are again obvious, and IV follows from the identity $U_{m} T_{n}-T_{n}=(n+1)^{-1} \sum_{0}^{m}\left(U_{m}-U_{j}\right)(n>m)$. To establish III' we employ the familiar expression $[34$, p. 44$]$

$$
T_{n} x=x(t)-\sigma_{n}(t)=x(t)-\frac{1}{\pi} \int_{-\pi}^{\pi} x(t+u) K_{n}(u) d u,
$$

where

$$
K_{n}(u)=\frac{1}{2(n+1)}\left[\frac{\sin (n+1) u / 2}{\sin (u / 2)}\right]^{2} \geqq 0,
$$

and $(1 / \pi) \int_{-\pi}^{\pi} K_{n}(u) d u=1$. It then follows trivially from the positiveness of the Fejér kernel $K_{n}(u)$ and the crudest of inequalities that $\left\|T_{n}\right\| \leqq 2$ for all $n$.

Hence $G$ is ergodic, and Fejér's theorem takes the form:

THEOREM 5.4. Every element of $C$ is ergodic under $G$ - for example $\lim _{n} T_{n} x$ $=0$ for every $x$ in $C$.

The following proof, although not elementary, is in keeping with our ap- 
proach: The integral representation above implies that the $T_{n} x(t)$ are uniformly bounded and equi-continuous, hence form a conditionally compact set. A strong limit point $y$ then exists, whence $y=\lim _{n} T_{n} x$ by Theorem 3.1, and $y=0$ since the null function is the only fix point.

In conclusion, we emphasize once again that ergodicity is a property of the semi-group $G$, rather than of the particular system $T_{\alpha}$ exhibited.

6. Quasi-compact operators. Conditions on the operators $T$ of $G$ sufficient to insure the ergodicity of every $x$ in $E$ are of interest. Yosida and Kakutani [33] found the special (bounded) case of Example 1 in which $T$ is quasicompact fundamental in their abstract treatment of Markoff processes.

Definition 6.1. A linear transformation $T$ is (weakly) compact $\left({ }^{7}\right)$ if the transform $T(S)$ of the unit sphere $S$ is conditionally (weakly) compact. $T$ is (weakly) quasi-compact if there exists an integer $m$ and a (weakly) compact linear transformation $V$ such that $\left\|T^{m}-V\right\|<1$.

THEOREM 6.1. If $G$ is ergodic and if some $T$ in $\bar{G}^{*}$ is (weakly) quasi-compact, then every $x$ in $E$ is ergodic. The projection $T_{\infty}$ is (weakly) compact, and the set $T_{\infty}(E)$ of fix points is a (reflexive) finite-dimensional subspace of $E$. If $T$ is quasi-compact and the limits IV hold uniformly for the system $T_{\alpha}$, then $T_{\infty}$ $=\lim _{\alpha} T_{\alpha}$ uniformly.

The generality of this theorem in comparison with the theorems of Yosida and Kakutani demands a corresponding simplification in proof. The basic tool is the identity

$$
T_{\alpha}=(I-D)^{-1} V T_{\alpha}+(I-D)^{-1}\left(T_{\alpha}-T^{m} T_{\alpha}\right)
$$

where $D=T^{m}-V$. Since $\|D\|<1$ by hypothesis, $(I-D)^{-1}=\sum_{0}^{\infty} D^{i}$ exists, and $(A)$ is readily verified. Recall also that the (weakly) compact operators form a two-sided ideal in the operator ring $B(E)$.

Given now any $x$ in $E$ we must produce a weak cluster point $y$ of $T_{\alpha} x$. Since $\lim _{\alpha}\left(T_{\alpha}-T^{m} T_{\alpha}\right) x=O$ (IVa), we need exhibit only a weak cluster point $y$ of the directed set $(I-D)^{-1} V T_{\alpha} x$. That such a $y$ exists follows from the (weak) compactness of the operator $(I-D)^{-1} V$ and the boundedness of the set $T_{\alpha} x$. Hence every $x$ is ergodic, and $T_{\infty}=\lim _{\alpha} T_{\alpha}$ strongly. Multiplying (A) on the right by $T_{\infty}$ and utilizing the equalities of Theorem 4.1, we obtain $T_{\infty}=T_{\alpha} T_{\infty}=(I-D)^{-1} V T_{\infty}$, whence $T_{\infty}$ is (weakly) compact.

We now show that if $T$ is quasi-compact and the limits IV hold uniformly for the system $T_{\alpha}$, then $T_{\infty}=\lim _{\alpha} T_{\alpha}$ uniformly. Setting $U=V(I-D)^{-1}$ and $R_{\alpha}=\left(T_{\alpha}-T_{\alpha} T^{m}\right)(I-D)^{-1}$, note first an identity dual to (A)

$$
T_{\alpha}=T_{\alpha} U+R_{\alpha} \text {. }
$$

$\left({ }^{7}\right)$ We prefer Dunford's terminology [10] to the usual term (weakly) completely continuous. Of particular interest in applications is the Dunford-Pettis [12] result: If $U, V$ in $B(L)$ are weakly compact, then $U V$ is compact. 
Similarly, we obtain $T_{\infty}=T_{\infty} T_{\alpha}=T_{\infty} U$. Since $\lim _{\alpha} R_{\alpha}=0$ uniformly, it is sufficient to show that $T_{\infty}=\lim _{\alpha} T_{\alpha} U$ uniformly-that is, $\lim _{\alpha}\left(T_{\infty}-T_{\alpha} U\right) x$ $=\lim _{\alpha}\left(T_{\infty}-T_{\alpha}\right) U x=0$ uniformly on $S$ or, equivalently, $\lim _{\alpha}\left(T_{\infty}-T_{\alpha}\right) x=0$ uniformly on $U(S)$. But the last assertion follows by standard techniques from the pointwise convergence of the equi-continuous $T_{\alpha}$ on the (conditionally) metrically compact set $U(S)$ : Given $\epsilon>0$, cover $U(S)$ with a finite number of spheres $S\left(y_{j} ; \epsilon / 3 M\right)$ with centers $y_{j}(j=1, \cdots, n)$ and radii $\epsilon / 3 M$. Choose an index $\beta$ such that $\alpha>\beta$ implies $\left\|T_{\infty} y_{j}-T_{\alpha} y_{j}\right\|<\epsilon / 3$ for all $j=1, \cdots, n$. That $\alpha>\beta$ implies $\left\|T_{\infty} x-T_{\alpha} x\right\|<\epsilon$ for every $x$ in $U(S)$ then follows from the inequality

$$
\left\|T_{\infty} x-T_{\alpha} x\right\| \leqq\left\|T_{\infty}\left(x-y_{J}\right)\right\|+\left\|T_{\infty} y_{J}-T_{\alpha} y_{J}\right\|+\left\|T_{\alpha}\left(y_{J}-x\right)\right\|
$$

on proper choice of $y_{J}$.

Since the set of fix points of $G$ is a closed linear subspace of $E$ equal to $T_{\infty}(E)$, the remaining assertions of the theorem are included in the following lemma, which is of independent interest.

LEMмA 6.1. Let $T$ be a (weakly) compact linear transformation of a Banach space $E$ into itself. Then every closed linear subspace $\Gamma$ contained in $T(E)$ is (reflexive) finite dimensional $\left({ }^{8}\right)$.

Proof. Write $E=\sum_{1}^{\infty} S_{j}$, where $S_{j}=[x \mid\|x\| \leqq j]$. Then $T(E)=\sum_{1}^{\infty} T\left(S_{j}\right)$ $\leqq \sum_{1}^{\infty} \bar{T}\left(S_{j}\right)$, the bar $(-)$ denoting the closure operation; and $\Gamma=\Gamma \cap T(E)$ $=\sum_{1}^{\infty} \Gamma \cap \bar{T}\left(S_{j}\right)$. Since $\Gamma$ is of the second category in itself and the $\Gamma \cap \bar{T}\left(S_{j}\right)$ are closed, one of the latter must contain a sphere of $\Gamma$. Since the $\Gamma \cap \bar{T}\left(S_{j}\right)$ are all (weakly) compact, the unit sphere of $\Gamma$ is (weakly) compact; and $\Gamma$ is respectively ([13], [2]) reflexive or finite dimensional.

7. The uniform ergodic theorem. The results of the last section suggest consideration of "uniform" ergodic theorems. Since $G \subset B(E)$ and every element of the Banach algebra $B(E)$ may be considered as an operator on $B(E)$, the norm as element and operator being the same, we may term $G$ "uniformly ergodic" if it is ergodic as a semi-group of operators on $B(E)$. It is clear that the characteristic properties of a "uniform system" of almost-invariant integrals then become: I: $T_{\alpha} \in B(E)$ for all $\alpha$; II: $T_{\alpha} \in \bar{G}^{*}$ for all $\alpha$ : III : $\left\|T_{\alpha}\right\| \leqq M$ for all $\alpha$; IV : for every $T$ in $G, \lim _{\alpha}\left\|T T_{\alpha}-T_{\alpha}\right\|=\lim _{\alpha}\left\|T_{\alpha} T-T_{\alpha}\right\|=0$. Moreover, it is apparent that the ergodicity of the element $I$ implies the ergodicity of every other element of $B(E)$. Hence the "uniform" ergodic theorem asserts that $\lim _{\alpha} T_{\alpha}=T_{\infty} \in B(E)$ exists uniformly under certain conditions.

Theorem 3.1 may now be formally translated into the terminology of the space $\varepsilon=B(E)$. We note, however, that the weak topology of $B(E)$ as a Banach space differs from what is commonly termed the "weak operator

$\left.{ }^{8}\right)$ Calkin $[7$, p. 401$]$ has shown that this property characterizes compact operators in Hilbert space. 
topology." Unfortunately, the latter topology appears more natural: The regularity of $E$ is equivalent to the compactness of the unit sphere $\mathfrak{U}_{1}$ of $B(E)$ in the weak topology as operators [8], while even in the case $E=$ Hilbert space $\mathfrak{U}_{1}$ is not well-behaved in the weak topology as elements.

The inherent peculiarities of the uniform ergodic theorem noted by Dunford [10] may be regarded as stemming from this distinction. It thus appears that the uniform ergodic theorem directly involves the algebraic structure of $B(E)$ and $G$ as well as topological properties of $E$ and the operators $T\left({ }^{9}\right)$. This fact is apparent even in the proof of Theorem 6.1 .

8. Ergodicity and its generalizations. We now consider briefly the problem of determining the ergodicity of a given semi-group $G$. That every bounded Abelian $G$ is (uniformly) ergodic appears to be the most general result available. Dropping the Abelian condition, one can find [1] a bounded $G$ such that some $\bar{O}(x)$ contains two fix points, whence $G$ is not ergodic. Taking $E$ as the real numbers, one can construct trivial examples of a non-ergodic unbounded Abelian $G$. The complexity of the question is finally apparent on extending Example 4 (\$5) to general Fourier expansions.

In the general non-Abelian case the existence of a system of almostinvariant integrals and the definition itself of an ergodic element are thus obscure. If $G$ is bounded, however, the ordering of $G^{*}$ in Example 3, although no longer necessarily of Moore-Smith type, suggests the following definition of ergodicity, introduced by Alaoglu and Birkhoff [1]:

Definition 8.1. If $G$ is bounded, an element $x$ of $E$ is ergodic if and only if the means $T^{*} x\left(T^{*} \in G^{*}\right)$ converge to a fix point $y$.

By convergence of the $T^{*} x$ to $y$ we mean: given $\epsilon>0$ and any $U$ in $G^{*}$ there exists $V \supset U$ such that $\|y-W x\|<\epsilon$ for all $W \supset V$. When $y$ is a fix point, the form $W=T^{*} V$ of the general successor of $V$ and the inequality $\|y-W x\|$ $=\| T^{*}(y-V x\|\leqq M\| y-V x \|$ imply that it is sufficient to find $V \supset U$ such that $\|y-V x\|<\epsilon$ for given $\epsilon>0$ and $U \in G^{*}$. Clearly, either formulation is equivalent to ordinary convergence of a direct set when the ordering of $G^{*}$ has the composition property, as in the Abelian case. More generally:

Theorem 8.1. If $G$ is bounded and ergodic, Definitions 3.1 and 8.1 of an ergodic element are equivalent.

Proof. The implication (8.1) $\rightarrow(3.1(1))$ is trivial. Conversely, given the $x, y$ of Theorem $3.1, \epsilon>0$, and $U$ in $G^{*}$, the invariance of the ergodic subspace $\Gamma$ expressed in the identity $y=T_{\infty} x=T_{\infty} U x$ implies the existence of $T^{*} \in G^{*}$ such that $\left\|y-T^{*} U x\right\|<\epsilon$. Now set $V=T^{*} U \supset U$. Hence (3.1) $\rightarrow(8.1)$.

REMARK. The following statements are easy corollaries of Definition 8.1 (cf. [1] and the proof of Lemma 4.1): If the $T^{*} x$ converge to an element $y$,

$\left(^{9}\right)$ Of course, the structure of $B(E)$ characterizes $E$ (Eidelheit's theorem [15]), but simple properties of $B(E)$ may translate in to complex properties of $E$ and conversely. 
then $y$ is unique and automatically a fix point. The ergodic elements again form a closed invariant subspace $\Gamma$. A necessary condition for ergodicity of $x$ is that $\bar{O}(x)$ contain a unique fix point; that $\bar{O}(x)$ contain a unique fix point for each element $x$ of an invariant set is a sufficient condition.

It turns out that restrictions on the space $E[1]$ or on the semi-group $G$ may reduce the ergodicity problem to establishing only the existence of a fix points in $\bar{O}(x)$. The requirement that $G$ be Abelian is an instance of the second type (Theorem 5.3). More generally, the following situation is of interest:

TheOREM 8.2. Let $G_{1}, G_{2}$ be bounded semi-groups that commute elementwise, and let every fix point of $G_{1}$ be a fix point of $G_{2}$, and conversely. Then

(1) The fix points of $\bar{O}_{1}(x)$ and $\bar{O}_{2}(x)$ reduce to a unique common fix point.

(2) If both $\bar{O}_{1}(x)$ and $\bar{O}_{2}(x)$ contain a fix point $y=T_{\infty} x$ for every $x$ in an invariant set $\Gamma$, then every $x$ of $\Gamma$ is ergodic under $G_{1}, G_{2}$, and $G=G_{1} G_{2}=G_{2} G_{1}$ with the limit fix point $y$.

Proof. We show first that if $y_{1} \in \bar{O}_{1}(x)$ and $y_{2} \in \bar{O}_{2}(x)$ are fix points, then $y_{1}=y_{2}$. Set $M=\max \left(M_{1}, M_{2}\right)$ and choose $T_{1}^{*} \in G_{1}^{*}, T_{2} \in G_{2}^{*}$ such that $\left\|y_{1}-T_{1}^{*} x\right\|<\epsilon / 2 M,\left\|y_{2}-T_{2}^{*} x\right\|<\epsilon / 2 M, \epsilon>0$ being arbitrary. Since $T_{1}^{*} T_{2}^{*}$ $=T_{2}^{*} T_{1}^{*}$ and $y_{1}, y_{2}$ are fix points under $G_{1}$ and $G_{2}$, we have

$$
\left\|y_{1}-y_{2}\right\|=\left\|T_{2}^{*}\left(y_{1}-T_{1}^{*} x\right)+T_{1}^{*}\left(T_{2}^{*} x-y\right)\right\|<\epsilon .
$$

Hence $y_{1}=y_{2}$.

Since $\bar{O}_{1}(x)$ and $\bar{O}_{2}(x)$ now contain at most one fix point, the asserted ergodicity of $x$ in (2) under $G_{1}$ and $G_{2}$ follows from the above Remark. It remains to prove the ergodicity of $x$ under $G=G_{1} G_{2}=G_{2} G_{1}$. Given $\epsilon>0$ and $T^{*}=\sum_{1}^{n} a_{j} U_{j} V_{j}$ in $G^{*}\left(a_{j} \geqq 0, \sum a_{J}=1, U_{j} \in G_{1}, V_{j} \in G_{2}\right)$ we must exhibit $a T^{* *}$ $\supset T^{*}$ in $G^{*}$ such that $\left\|y-T^{* *} x\right\|<\epsilon$.

Since $x$ is ergodic under $G_{1}$ we can choose in succession $T_{1}^{*}, \cdots, T_{n}^{*}$ in $G_{1}^{*}$ such that

$$
\left\|T_{j}^{*} T_{j-1}^{*} \cdots T_{1}^{*} U_{j} x-y\right\|<\epsilon / M^{2} .
$$

Then $\left\|T_{j}^{*} \ldots T_{1}^{*} U_{j} V_{j} x-y\right\|=\left\|V_{j}\left(T_{j}^{*} \cdots T_{1}^{*} U_{j} x-y\right)\right\|<\epsilon / M$. Setting $T^{* *}$ $=T_{n}^{*} \cdots T_{1}^{*} T^{*} \supset T^{*}$, we find

$$
\begin{aligned}
\left\|T^{* *} x-y\right\| & =\left\|\sum a_{j} T_{n}^{*} \cdots T_{j+1}^{*}\left(T_{j}^{*} \cdots T_{1}^{*} U_{j} V_{j} x-y\right)\right\| \\
& <\sum a_{j} M \cdot \epsilon / M=\epsilon .
\end{aligned}
$$

9. Almost periodic functions. We conclude our discussion of ergodicity by sketching a proof of the von Neumann [25] mean value theorem for almost periodic functions defined on arbitrary groups. Our aim is to subsume the mean value theorem under the generalized ergodic theory. 
Given an arbitrary group $G$, denote by $M(G)$ the space of bounded real- or complex-valued functions $x(t)(t \in G)$ under the norm $\|x\|=\sup _{t \in G}|x(t)| . G$ is then faithfully represented as the group of left translations $G_{L}: U_{a} x(t)$ $=x\left(a^{-1} t\right)$, or as the group of right translations $G_{R}: V_{a x} x(t)=x(t a)$. Since $\left\|U_{a}\right\|=\left\|V_{a}\right\|=1$, the only fix points are the constant functions, and the elementwise commutativity of $G_{L}$ and $G_{R}$ follows from the associativity of group multiplication, the preliminary conditions of Theorem 8.2 are fulfilled.

By definition $x(t) \in M(G)$ is left (right) almost periodic if the set of translates $\left[U x \mid U \in G_{L}\right]\left(\left[-V x \mid V \in G_{R}\right]\right)$ is conditionally compact. We recall that Maak [24] has proved the equivalence of left and right almost periodicity, and that the almost periodic subspace $\Gamma$ is a closed invariant linear subspace of $M(G)$ (Theorem 4.2). Hence to establish ergodicity we must show that $\bar{O}_{L}(x)$ or $\bar{O}_{R}(x)(x \in \Gamma)$ contains a fix point.

Given $x(t)$ in $\Gamma$ consider finite decompositions of $G_{L}$ into disjoint sets, $G_{L}=\sum_{1}^{m} G_{j}$, such that for any $U_{1}, U_{2}$ in $G_{j}\left\|U_{1} x-U_{2} x\right\|<\epsilon$. The existence of at least one such decomposition follows from the conditional compactness of $\left[U x \mid U \in G_{L}\right]$. Now choose a minimal decomposition-that is, one with the least number of elements-and set

$$
T_{\epsilon}=\frac{1}{m} \sum_{1}^{m} T_{j}
$$

The $T_{\epsilon}$ thus defined is not unique. If under the same decomposition one sets

$$
T^{\prime}=\frac{1}{m} \sum_{1}^{m} T_{j}^{\prime}
$$

it is clear that $\left\|T_{\epsilon} x-T_{\epsilon}^{\prime} x\right\|<\epsilon$. What is crucial, however, is that if one employs another minimal $\epsilon$-decomposition $G_{L}=\sum_{1}^{m} G_{j}^{\prime}$, a general combinatorial lemma of Maak [24] asserts that one can pair off the $G_{j}$ with the $G_{j}^{\prime}$ in such fashion that corresponding sets have a non-vacuous intersection. It follows that if $T_{\epsilon}, T_{\epsilon}^{\prime}$ are any two means arising from $\epsilon$-minimal decompositions, then $\left\|T_{\epsilon} x-T_{\epsilon}^{\prime}\right\| x<2 \epsilon$.

Now order the $T_{\epsilon}$ in the obvious fashion: $T_{\epsilon} \supset T_{\epsilon^{\prime}}$ means $\epsilon<\epsilon^{\prime}$. The directed system thus obtained obviously satisfies I, II, III of Definition 2.1. Moreover, IV(a) holds: To see this, note that $T \in G_{L}$ implies $G_{L}=T G_{L}$ $=\sum_{1}^{m} T G_{j}$ is again a minimal $\epsilon$-decomposition, and $T T_{\epsilon}=(1 / m) \sum_{1}^{m} T T_{j}$ is the corresponding mean. Hence $\left\|T_{\epsilon} x-T T_{\epsilon} x\right\|<2 \epsilon$ by the above remark.

The validity of I, II, III, and of IV(a) at the point $x$, plus the metric compactness of $\bar{O}_{L}(x)$ are sufficient, as noted in $\$ 3$, to insure the existence of a fix point in $\bar{O}_{L}(x)$. A fix point in $\bar{O}_{R}(x)$ is similarly obtained, and the common fix point is a constant function-the mean value of $x$. All conditions of Theorem 8.2 are now fulfilled, whence we have:

Theorem 9.1 (Mean Value Theorem). Every almost periodic $x(t)$ in 
$M(G)$ is ergodic under $G_{L}, G_{R}$, and $G_{L} G_{R}$.

On setting $E=\Gamma$ we note that $T_{\infty} \in B(E),\left\|T_{\infty}\right\|=1$, and $T_{\infty}=T T_{\infty}=T_{\infty} T$ for all $T \in G_{L} G_{R}$. $G_{L}$ or $G_{R}$ is then ergodic by hindsight since $T_{\infty}$ itself may be taken as the system $T_{\alpha}$.

Since every continuous function on a compact group is almost periodic, the existence of the invariant integral mean for compact groups is, of course, a special case. Haar measure can then be obtained from the invariant integral by standard devices. A characteristic (advantage or disadvantage?) of this approach is that the measure obtained is automatically normalized-that is, $m(G)=1$.

\section{PART II. WEAK ALMOST PERIODIC FUNCTIONS}

10. Introduction. We consider henceforth a locally compact Abelian topological group $G$ (Weil [32]) and the space $C(G)$ of bounded complex-valued continuous functions $x(t)$ on $G$ under the norm $\|x\|=\sup _{t \in G}|x(t)|$. Since $G$ is faithfully represented as a bounded Abelian group of translations on $C(G)-U_{a} x(t)=x(t+a)$ - the considerations of Examples 2 and 3 ( $\$ 5$ ) suggest a further study of the class of weak almost periodic functions:

Definition $10.1 x(t) \in C(G)$ is weakly almost periodic (w.a.p.) if the set of translates $[x(t+s) \mid s \in G]$ is conditionally weakly compact. $\mathfrak{B}$ denotes the class of w.a.p. functions.

Two factors complicate our discussion of the class $\mathfrak{W}$ and distinguish it from a purely formal generalization of the standard theory of almost periodic functions. We note first that the operators $U_{a} x(t)=x(t+a)$, although equicontinuous in the norm topology of $C(G)$, are not equi-continuous in the weak topology. The Bochner and Bohr definitions [18] of almost periodicity split apart; and our theory differs from the theory of almost periodic points of transformation groups based on the latter definition. However, we find that this lack of equi-continuity is compensated to some extent-for example the existence of a mean value-by the commutativity of $G$.

The second and more serious complication lies in the obscure structure of the weak topology of $C(G)$. Even in the case $G=R_{1}$ (additive group of the reals) no simple or convenient representation for the general linear functional is at hand. Determining the weak almost periodicity of a given function thus involves some special device to circumvent this difficulty. The fact that we can limit ourselves to sequential weak compactness notions (Theorem 1.2) provides a crucial simplification. Even sequential weak convergence, however, is refractory: For example, the sequence $x_{n}(t)=\exp (i t / n)$ does not converge weakly to 1 in $C\left(R_{1}\right)$, despite the fact that $\lim _{n} x_{n}(t)=1$ uniformly on every finite interval and $\left\|x_{n}\right\|=1$ for all $n\left({ }^{10}\right)$.

11. Examples of w.a.p. functions. It is trivial that every almost periodic

(10) If $f(x)$ denotes some extension of the mean value (of a.p. functions) to a linear functional on $C\left(R_{1}\right)$, then $f\left(x_{n}\right)=0$ for all $n$, but $f(1)=1$. 
function is weakly almost periodic. More interesting examples are continuous functions vanishing at infinity and positive definite functions (hence FourierStieltjes transforms). In both cases the fundamental difficulty mentioned above is bypassed by a special device.

THEOREM 11.1. A continuous function $x(t)$ vanishing at infinity is weakly almost periodic-that is, $C_{0}(G) \subset \mathfrak{B}$.

Proof. Since $C_{0}(G)$ is a closed linear subspace of $C(G)$, and weak sequential convergence in $C_{0}(G)$ is equivalent $(\$ 1)$ to pointwise convergence and boundedness, we must show that given any sequence $y_{n}(t)=x\left(t+s_{n}\right)$ of translates of $x(t) \in C_{0}(G)$, there exists a subsequence $y_{n_{j}}(t)$ and $z(t) \in C_{0}(G)$ such that $\lim _{n} y_{n_{j}}(t)=z(t)$ for all $t$ in $G$. The boundedness condition is, of course, trivially satisfied.

Since the $y_{n}(t)$ vanish at infinity we can find compact sets $\Gamma_{n, i} \subset G$ such that $t \in G-\Gamma_{n, i}$ implies $\left|y_{n}(t)\right|<1 / i$. Setting $\Gamma=\sum_{n, i=1}^{\infty} \Gamma_{n, i}$, we obtain $y_{n}(t)=0$ for all $n$ if $t \in G-\Gamma$. Since each $\Gamma_{n, i}$ is a compact uniform space and the $y_{n}(t)$ are uniformly bounded and equi-continuous, we can [18] pick out a subsequence $y_{n_{i}}(t)$ such that $y_{n_{i}}(t)$ converges uniformly on $\Gamma_{n, i}$. The diagonal process then yields a final subsequence $y_{n_{j}}(t)$ and a function $z(t)$ such that $\lim _{n} y_{n}(t)=z(t)$ for all $t \in \Gamma$; and the limit holds for all $t$ on setting $z(t)=0$, $t \in G-\Gamma\left({ }^{11}\right)$.

It remains to prove that $z(t) \in C_{0}(G)$. There are two cases: (1) There exists a compact set $\Sigma$ containing an infinite number of the $s_{n_{j}}$; (2) no compact set contains more than a finite number of the $s_{n_{j}}$. In the first case the $s_{n_{j}}$ in $\Sigma$ possess a cluster point $s$, whence it follows readily that $z(t)=x(t+s) \in C_{0}(G)$. In the second case it is clear that $z(t) \equiv 0$.

DEFINITION 11.1. $x(t)$ is positive definite if it is continuous and if it satisfies

$$
\sum_{i, j} \alpha_{i} \bar{\alpha}_{j} x\left(s_{j}-s_{i}\right) \geqq 0
$$

for an arbitrary finite set of complex numbers $\alpha_{i}$ and elements $s_{i} \in G$.

Theorem 11.2. A positive definite function on $G$ is weakly almost periodic.

Proof. For the properties of positive definite functions see Bochner [4], Weil [32], Raikov [27], and Godement [17]. The only property we use is the characteristic correspondence [17] between positive definite functions and strongly continuous unitary representations $t \rightarrow U_{t}$ of $G$ in Hilbert space $\mathfrak{S}$, expressed in the relation

$$
x(t)=\left(X, U_{t} X\right) \quad(X \in \mathfrak{S})\left({ }^{12}\right) .
$$

(11) We would effectively omit the argument of this paragraph if we imposed the second countability axiom on $G$.

(12) The equivalence of the theorems of Stone and Bochner [32] is contained in this correspondence. 
More generally, given $Y \in \mathfrak{S}$ consider the linear transformation $T X=x$ of $\mathfrak{S}$ into $C(G)$ defined by the relation $x(t)=\left(X, U_{t} Y\right)$. Clearly, $\|x\| \leqq\|X\|$ - $\left\|U_{t} Y\right\|=\|X\| \cdot\|Y\|$, whence $\|T\| \leqq\|Y\|$. We now derive the weak almost periodicity of every $x(t)$ arising in this fashion from the weak compactness of the unit sphere of $\mathfrak{S}$.

Recalling that the adjoint transformation $T^{*} f=F$, where $F(X)=f(T X)$, maps $C(G)^{*}$ into $\mathfrak{S}^{*}=\mathfrak{E}$, consider any sequence of translates

$$
x\left(t+s_{n}\right)=\left(X, U_{t+s_{n}} Y\right)=\left(U_{s_{n}}^{-1} X, U_{t} Y\right)=T U_{s_{n}}^{-1} X .
$$

Since $\left\|U_{s_{n}}^{-1} X\right\|=\|X\|$ for all $n$, there exists a subsequence $s_{n}^{\prime}$ such that $\lim _{n} U_{s_{n}^{\prime}}^{-1} X=X_{0}$ weakly for some $X_{0}$ in $\mathfrak{S}$. Set $x_{0}(t)=T X_{0}$. That $\lim _{n} x\left(t+s_{n}^{\prime}\right)$ $=x_{0}(t)$ weakly in $C(G)$ then follows from the above remark-that is, from the weak continuity of $T$. Hence $x(t)$ is w.a.p.

It is apparent that the above proof generalizes to yield

THEOREM 11.3. If $t \rightarrow U_{t}$ is a strongly continuous representation of $G$ in a group of uniformly bounded linear transformations in a Banach space $E$, if $f \in E^{*}$, and if $\left[U_{t} X \mid t \in G\right]$ is conditionally weakly compact for some $X \in E$, then $x(t)=f\left(U_{t} X\right)$ is weakly almost periodic.

That the general w.a.p. function is of this type follows trivially on setting $E=\mathfrak{W}, X=x(t), \quad U_{a} X=x(t+a)$, and $f(X)=x(0)$ (Theorems 12.1 and 13.1 infra).

Since the faithful representation of $G$ in $L^{p}(G)(p \geqq 1)$ is strongly continuous $[32$, p. 41$]$, we obtain

Corollary 11.3.1. If $f(s) \in L^{p}(G), g(s) \in L^{q}(G),\left(p>1, p^{-1}+q^{-1}=1\right)$, then $x(t)=\int_{G} f(t+s) g(s) d s$ is weakly almost periodic.

12. $\mathfrak{W}$ as a Banach $*$-algebra. We now examine the algebraic structure of the space $\mathfrak{B}$ of w.a.p. functions.

THEOREM 12.1. $\mathfrak{W}$ is an invariant $B^{*}$ subalgebra of $C(G)$-that is,

(1) $\mathfrak{W}$ is a closed linear subspace.

(2) $x(t) \in \mathfrak{W}$ implies $x(t+s) \in \mathfrak{W}$.

(3) $1 \in \mathfrak{W}$.

(4) $x(t) \in \mathfrak{W}$ implies (a) $x(-t) \in \mathfrak{W} ;(b) \bar{x}(t) \in \mathfrak{W}$.

(5) If $x(t), y(t)$ are in $\mathfrak{W}$, then $x(t) y(t)$ is in $\mathfrak{W}$.

(6) $x(t) \in \mathfrak{W}$ implies $|x(t)|^{p} \in \mathfrak{W}(p \geqq 0)$.

Proof. (1) and (2) are a specialization of Theorem 4.2. (3) and (4a) are trivial, and (4b) follows from the almost obvious fact that $\lim _{n} x_{n}(t)=x(t)$ weakly in $C(G)$ implies $\lim _{n} \bar{x}_{n}(t)=\bar{x}(t)$ weakly. The proof of the seemingly elementary assertion (5), however, is surprisingly deep: Not only does the reduction to sequential compactness appear an absolute necessity, rather than a convenience, but one is forced to go "outside" the space $C(G)$. 
Given $x(t), y(t)$ in $\mathfrak{W}$ and any sequence $\left(s_{n}\right)$ in $G$, choose a subsequence $\left(s_{n}^{\prime}\right)$ and elements $X(t), Y(t)$ in $C(G)$ such that $\lim _{n} x\left(t+s_{n}^{\prime}\right)=X(t)$ weakly, $\lim _{n} y\left(t+s_{n}^{\prime}\right)=Y(t)$ weakly. To conclude that $\lim _{n} x\left(t+s_{n}^{\prime}\right) y\left(t+s_{n}^{\prime}\right)$ $=X(t) Y(t)$ weakly, we must establish

LemmA 12.1. If $x=\lim _{n} x_{n}$ weakly, $y=\lim _{n} y_{n}$ weakly in $C(G)$, then $x y=\lim _{n} x_{n} y_{n}$ weakly.

If $G$ were compact, weak sequential convergence would reduce to pointwise convergence and boundedness (Theorem 1.3), and the lemma would follow immediately. In the general case we therefore represent $C(G)$ as the ring $C(\Omega)$ of all complex-valued continuous functions on a compact Hausdorff space $\Omega: x(t) \leftrightarrow X(\tau) ;\|x\|=\|X\|=\sup _{\tau \in \Omega}|X(\tau)| . \Omega$ may be regarded as the space of maximal ideals of the $B^{*}$ algebra $C(G)$ produced by the Stone-Gelfand-Neumark theory [16], or simply as the Cech compactification of the completely regular space $G$. The isomorphism $x \leftrightarrow X$ being norm preserving, the validity of the lemma in $C(\Omega)$ implies the validity in $C(G)$.

Since $\mathfrak{B}$ itself may now be regarded as a $C\left(\Omega^{\prime}\right),|x(t)|=[x(t) \bar{x}(t)]^{1 / 2}$ is defined in $C\left(\Omega^{\prime}\right)$ and hence in $\mathfrak{W}$. $|x(t)|^{p}(p \geqq 0)$ exists similarly, and the proof is complete.

13. Uniform continuity of w.a.p. functions. We have made no explicit. use, as yet, of the local compactness of $G$. We now show that the existence and absolute continuity of Haar measure on $G$ imply the uniform continuity of w.a.p. functions. Uniform continuity itself will be required later to establish an analogue of the Fubini theorem for mean values.

THEOREM 13.1. A weakly almost periodic function is uniformly continuous.

Proof. We assume $x(t) \in \mathfrak{W}$ is not uniformly continuous and derive a contradiction. By hypothesis, there is an $\epsilon>0$ such that given any (symmetric) nbd. $N(0)$ in $G$ there exist $u, v$ in $G$ such that $u-v \in N(0)$ but $|x(u)-x(v)| \geqq \epsilon$. We can thus obtain in standard fashion a directed set $\left(u_{\alpha}, v_{\alpha}\right) \subset G \times G$ such that (1) $\lim _{\alpha}\left(u_{\alpha}-v_{\alpha}\right)=0 ;(2)\left|x\left(u_{\alpha}\right)-x\left(v_{\alpha}\right)\right| \geqq \epsilon$ for all $\alpha$. Set $z_{\alpha}(t)=x\left(t+u_{\alpha}\right)$ $-x\left(t+v_{\alpha}\right)$. Since $x(t)$ is w.a.p., the set of functions $z_{\alpha}(t)$ possesses a weak cluster point $w(t)$. Since $\left|z_{\alpha}(0)\right| \geqq \epsilon$ for all $\alpha$ by (2), we must have $|w(0)| \geqq \epsilon>0$. We now obtain the desired contradiction by establishing $w(t) \equiv 0$.

It is clearly sufficient to prove that $\int_{\Gamma} w(t) d t=0$ for every compact set $\Gamma$ in $G$. Since $w(t)$ is a weak cluster point of $z_{\alpha}(t)$ and $f(x)=\int_{\Gamma} x(t) d t$ is a linear functional on $C(G)$, we need only show that $\lim _{\alpha} \int_{\Gamma} z_{\alpha}(t) d t=0$. Now

$$
\begin{aligned}
\left|\int_{\Gamma} z_{\alpha}(t) d t\right| & =\left|\int_{\Gamma}\left\{x\left(t+u_{\alpha}\right)-x\left(t+v_{\alpha}\right)\right\} d t\right| \\
& =\left|\left\{\int_{\Gamma_{+u_{\alpha}}}-\int_{\Gamma_{+v_{\alpha}}}\right\} x(t) d t\right| \leqq\|x\| \cdot m\left(\Delta_{\alpha}\right),
\end{aligned}
$$


where $\Delta_{\alpha}$ is the symmetric difference of the sets $\Gamma+u_{\alpha}$ and $\Gamma+v_{\alpha}$. The invariance and absolute continuity of Haar measure [27] in conjunction with condition (1) imply that $\lim _{\alpha} m\left(\Delta_{\alpha}\right)=0$; and the proof is complete.

14. The mean value. Since every element $x$ of $\mathfrak{B}$ is ergodic under the group $G$ operating on $C(G)$ (Theorem 5.3), to every $x \in \mathfrak{W}$ corresponds a fix point or constant function $T_{\infty} x$. The numerical value $M_{s} x(s)=T_{\infty} x$ is then the mean value of $x(s)$. Since the characteristic property of $T_{\infty} x$ is that it can be approximated arbitrarily closely in $C(G)$ by convex combinations of translates of $x(s), M_{s} x(s)$ coincides with the von Neumann [25] mean value. Regarded as a functional on $\mathfrak{B}$, the characteristic properties of $M_{s}$ are the following:

THEOREM 14.1. If $x(s)$ and $y(s)$ are w.a.p. all the functions $x(t+s), x(-s)$, $\bar{x}(s),|x(s)|,|x(s)|^{2}, x(s) y(s), a x(s) \pm b y(s)(a, b$ complex numbers; $t \in G$ ) are w.a.p. (cf. Theorem 12.1). Furthermore:

(1) $M_{s}[a x(s) \pm b y(s)]=a M_{s} x(s) \pm b M_{s} y(s)$.

(2) $M_{s} 1=1$.

(3) $x(s) \geqq 0$ for all $s \in G$ implies $M_{s} x(s) \geqq 0$.

(4) $\left|M_{8} x(s)\right| \leqq M_{s}|x(s)| \leqq\|x\|$.

(5) $M_{s} \bar{x}(s)=\bar{M}_{s} x(s)$.

(6) $M_{8} x(s+t)=M_{8} x(s)$.

(7) $M_{8} x(-s)=M_{s} x(s)$.

(8) $\left|M_{s}\{x(s) y(s)\}\right| \leqq\left[M_{s}\left\{|x(s)|^{2}\right\}\right]^{1 / 2} \cdot\left[M_{s}\left\{|y(s)|^{2}\right\}\right]^{1 / 2}$.

Conversely, the formal properties (1)-(8) determine $M_{8} x(s)$ uniquely-in fact, any $M_{8} x(s)$ satisfying (1)-(3) and (6) must equal $T_{\infty} x$.

Since these assertions are elementary consequences of the above definition of mean value and the von Neumann [25] derivation for almost periodic functions requires no modification, we omit the proof. We emphasize at this point, however, a vital difference between almost periodic and weak almost periodic functions: If $x(t)$ is almost periodic a stronger form of (3) is validthat is, $M_{8}|x(s)| \geqq 0$, the equality holding if and only if $x(t) \equiv 0$. This property fails for weak almost periodic functions. For example, if $G=R_{1}$ and $x \in \mathfrak{W} \cap L(-\infty, \infty)$ it is clear ( $\$ 5$, Example 2) that $M_{s}|x(s)|$ $=\lim _{\alpha \rightarrow \infty} \alpha^{-1} \int_{0}^{\alpha}|x(s)| d s=0$. More generally, if $x(t) \in \mathfrak{W}$ vanishes at infinity, we find that $M_{t}|x(t)|=0$.

Following von Neumann we now consider w.a.p. functions on the product group $G^{2}=G \times G$ and establish a Fubini theorem for the mean value.

THEOREM 14.2. If $x(t) \in \mathfrak{W}(G), x(t)$ and $x(t \pm s)$ are in $\mathfrak{W}\left(G^{2}\right)$. Conversely, if $x(s, t) \in \mathfrak{W}\left(G^{2}\right), x(s, t)$ is weakly almost periodic as a function of $s$ or $t$. $M_{8} x(s, t), M_{t} x(s, t)$ then exist and are weakly almost periodic in the remaining variable, and

$$
M_{t}\left\{M_{s} x(s, t)\right\}=M_{s}\left\{M_{t} x(s, t)\right\}=M_{s, t} x(s, t) .
$$


Proof. The first two assertions are obvious. Since $s, t$ appear symmetrically it is sufficient to consider only $M_{s} x(s, t)$-as a function of $t$-where $x(s, t)$ $\in \mathfrak{W}\left(G^{2}\right)$. The boundedness of $M_{s} x(s, t)$ is evident and continuity follows from the uniform joint continuity (Theorem 13.1) of $x(s, t)$ : Given $\epsilon>0$ there exists a (symmetric) nbd. $N(0)$ such that $t_{1}-t_{2} \in N(0)$ implies $\left|x\left(s, t_{1}\right)-x\left(s, t_{2}\right)\right|$ $<\epsilon$ for all $s$, whence $\left|M_{s}\left[x\left(s, t_{1}\right)-x\left(s, t_{2}\right)\right]\right|<\epsilon$. Hence $M_{s} x(s, t)$ is in $C(G)$.

That $M_{s} x(s, t)$ is w.a.p. in $t$ follows from the fact that the linear mapping $x(s, t) \rightarrow M_{s} x(s, t)$ of $\mathfrak{W}\left(G^{2}\right)$ into $C(G)$ is strongly, and hence weakly, continuous. Since $M_{t}\left[M_{s} x(s, t)\right]$ now exists and clearly has the properties (1)-(3) and (6) (Theorem 14.1) of the double mean, it must coincide with the latter by the uniqueness theorem-that is, $M_{t}\left\{M_{s} x(s, t)\right\}=M_{s, t} x(s, t)$. Interchanging $s$ and $t$ we obtain the remaining assertions, and the proof is complete.

15. Fourier expansions of w.a.p. functions. We consider now the Fourier analysis of w.a.p. functions and obtain the Parseval equation as our final result, the analogue of the approximation theorem, if any, for w.a.p. functions remaining open. The procedure combines the methods of von Neumann [25] and Bochner [4], the crucial fact being that the convolution of two w.a.p. functions is almost periodic.

DEFINITION 15.1. If $x(t)$ and $y(t)$ are w.a.p., the convolution $z=x * y$ is defined by $z(t)=M_{s}[x(t-s) y(s)]=M_{s}[x(s) y(t-s)]$.

The equivalence of the two expressions for $z(t)$ follows from (6) and (7) of Theorem 14.1 on replacing $s$ by $t-s$, and $z(t)$ is w.a.p. by Theorem 14.2. One may then show that $\mathfrak{W}$ forms a commutative ring under (pointwise) addition and the new "multiplication" $x * y$, the associativity of "multiplication" following from an interchange of mean values justified by Theorem 14.2. More to the point, however, is

Theorem 15.1. If $x(t)$ and $y(t)$ are weakly almost periodic, then $z=x * y$ is almost periodic $\left({ }^{13}\right)$.

Proof. Let $\left(u_{n}\right)$ be any sequence in $G$. Then $z\left(t+u_{n}\right)=M_{8}\left[x(s) y\left(t+u_{n}-s\right)\right]$ $=M_{s}\left[x\left(s+u_{n}\right) y(t-s)\right]$, on replacing $s$ by $s+u_{n}$. Since $x(t)$ is w.a.p. there exists a subsequence $\left(u_{n}^{\prime}\right)$ and $X \in \mathfrak{W}$ such that $\lim _{n} x\left(s+u_{n}^{\prime}\right)=X(s)$ weakly. We now show that $\lim _{n} z\left(t+u_{n}^{\prime}\right)=M_{s}[X(s) y(t-s)]$ uniformly in $t$.

The Schwartz inequality (Theorem 14.1 (8)) implies

$$
\begin{aligned}
\mid z\left(t+u_{n}{ }^{\prime}\right)-M_{s}[X(s) & y(t-s)]\left.\right|^{2} \\
& =\left|M_{s}\left[\left\{x\left(s+u_{n}{ }^{\prime}\right)-X(s)\right\} y(t-s)\right]\right|^{2} \\
& \leqq M_{s}\left[\left|X(s)-x\left(s+u_{n}^{\prime}\right)\right|^{2}\right] \cdot M_{s}\left[|y(t-s)|^{2}\right] \\
& \leqq\|y\|^{2} \cdot M_{s}\left[\left|X(s)-x\left(s+u_{n}^{\prime}\right)\right|^{2}\right] .
\end{aligned}
$$

The last expression not involving $t$, the assertion follows from the remark

$\left({ }^{13}\right)$ Godement [17] obtains an analogous result for a not necessarily Abelian group, but for more special functions-for example, $x(t)=y(t)$ positive definite. 
that $\lim _{n}\left|X(s)-x\left(s+u_{n}^{\prime}\right)\right|^{2}=0$ weakly (cf. $\left.\$ 12\right), M_{s}$ being a linear functional on $\mathfrak{B}$.

We now introduce the characters of $G$-that is, continuous complexvalued functions $\lambda(t)=(t, \lambda)$ on $G$ such that $(1)\left(t_{1}+t_{2}, \lambda\right)=\left(t_{1}, \lambda\right)\left(t_{2}, \lambda\right) ;(2)$ $|(t, \lambda)|=1$ for all $t \in G$. The characters $\lambda$ form an additive Abelian group $G^{*}$ on setting $(t, 0) \equiv 1,\left(t, \lambda_{1}+\lambda_{2}\right)=\left(t, \lambda_{1}\right)\left(t, \lambda_{2}\right)$, and $(t,-\lambda)=(t, \lambda)^{-1}=(-t, \lambda)$ $=\bar{\lambda}(t)$. It is trivial that a character is almost periodic and that $M_{s}(s, \lambda)=0$ if $\lambda \neq 0$.

Consider the formal Fourier expansion

$$
x(t) \sim \sum_{\lambda \in G^{*}} a(\lambda)(t, \lambda)
$$

$(x \in \mathfrak{W})$,

where $a(\lambda)=M_{s}[x(s)(s,-\lambda)]$. If $a(\lambda) \neq 0$, we call $\lambda$ a "proper exponent" of $x(t)$. Exactly as in the ordinary case [3] we obtain

LEMMA 15.1. If $\left(\lambda_{1}, \cdots, \lambda_{N}\right)$ is any finite set of proper exponents of $x(t)$ $\in \mathfrak{W}$, then

$$
0 \leqq M_{8}\left[\left|x(s)-\sum_{1}^{N} a\left(\lambda_{n}\right)\left(s, \lambda_{n}\right)\right|^{2}\right]=M_{8}|x(s)|^{2}-\sum_{1}^{N}\left|a\left(\lambda_{n}\right)\right|^{2} .
$$

Consequently, $\sum_{1}^{N}\left|a\left(\lambda_{n}\right)\right|^{2} \leqq M_{8}|x(s)|^{2}$. Hence the set of proper exponents is at most countable-say $\left(\lambda_{n}\right)$ - and

$$
\sum\left|a\left(\lambda_{n}\right)\right|^{2} \leqq M_{8}|x(s)|^{2}<\infty \quad \text { (Bessel's Inequality). }
$$

LEMmA 15.2. If $x(t) \in \mathfrak{W}$ let $z(t)=M_{s}[x(s) \bar{x}(s-t)]=x * \bar{x}$, where $\tilde{x}(t)$ $=\bar{x}(-t)$. If $(t, \lambda)$ is any character, then

$$
c(\lambda)=M_{s}[z(s)(s,-\lambda)]=\mid M_{s}\left[\left.x(s)(s,-\lambda]\right|^{2}=|a(\lambda)|^{2} .\right.
$$

Proof. $\quad c(\lambda)=M_{s}[z(s)(s,-\lambda)]=M_{s}\left[M_{u}\{x(u) \bar{x}(u-s)\}(s,-\lambda)\right]$ $=M_{u}\left[M_{8}\{\bar{x}(u-s)(s, \quad-\lambda)\} x(u)\right]=M_{u}\left[x(u)(u, \quad-\lambda) M_{8}\{\bar{x}(-s)(s,-\lambda)\}\right]$ $=M_{u}[x(u)(u,-\lambda)] \cdot M_{s}[\bar{x}(s)(-s,-\lambda)]=a(\lambda) \cdot \bar{a}(\lambda)=|a(\lambda)|^{2}$, the inversion of mean values being justified by Theorem 14.2 , the other operations by Theorem 14.1 and the definition of characters.

Consider finally the expansion of the almost periodic function $z=x * \tilde{x}$ : $z(t) \sim \sum c(\lambda)(t, \lambda)$. Since $c(\lambda)=|a(\lambda)|^{2}, z(t)$ and $x(t)$ have the same proper exponents $\left(\lambda_{n}\right)$. Moreover, (B) and the uniqueness theorem for almost periodic functions [32] imply that we can write $z(t)=M_{s}[x(s) \bar{x}(s-t)]$ $=\sum\left|a\left(\lambda_{n}\right)\right|^{2}\left(t, \lambda_{n}\right)$, the series on the right being absolutely and uniformly convergent. Setting $t=0$, we obtain

TheOREM 15.2 (PARSEVAL's EquATION). If $x(t) \in \mathfrak{B}$ write formally $x(t)$ $\sim \sum a(\lambda)(t, \lambda)$, where $a(\lambda)=M_{s}[x(s)(s,-\lambda)]$. Then $a(\lambda) \neq 0$ for at most $a$ countable set of characters $\left(\lambda_{n}\right)$-the proper exponents-and 


$$
M_{8}|x(s)|^{2}=\sum\left|a\left(\lambda_{n}\right)\right|^{2}
$$

From this theorem and Lemma 15.1 (A) follows

Corollary 15.2.1 (Mean Convergence).

$$
\lim _{N \rightarrow \infty} M_{8}\left[\left|x(s)-\sum_{1}^{N} a\left(\lambda_{n}\right)\left(s, \lambda_{n}\right)\right|^{2}\right]=0 .
$$

16. W.a.p. functions on the line. We conclude our discussion of w.a.p. functions by considering the relation of the class $\mathfrak{W}$ to other classes of generalized almost periodic functions in the special case $G=R_{1}$. We recall first (Example $3, \S 5)$ that $M_{t} x(t)=\lim _{L \rightarrow \infty}(1 / L) \int_{s}^{s+L} x(t) d t \quad(x \in \mathfrak{B})$, the convergence being uniform in $s$. Now the Weyl norm is defined $[3 ; 5]$ as

$$
\|x\|_{W^{p}}=\left[\lim _{L \rightarrow \infty} \sup _{-\infty<s<\infty} \frac{1}{L} \int_{s}^{s+L}|x(t)|^{p} d t\right]^{1 / p} \quad(p \geqq 1),
$$

the limit existing, in particular, if $x(t)$ is bounded and measurable. If

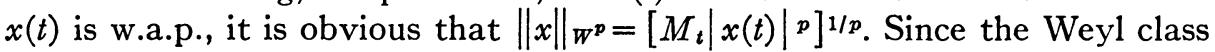
$W^{p}$ of generalized a.p. functions consists, by definition, of functions $x(t)$ that are limits in the $W^{p}$ metric of finite linear combinations of the characters $\exp (i \lambda t)$, the corollary above implies that a w.a.p. function $x(t)$ lies in the class $W^{2}$. Consequently, since $x(t)$ is bounded, $x$ lies in $W^{p}$ for every $p \geqq 1$ [cf. 5, p. 62].

The relation of $\mathfrak{W}$ to the Stepanoff class $S^{p}(p \geqq 1)$ is implicit in the following theorem of Bochner [3]: A uniformly continuous $S^{p}$ a.p. function is almost periodic.

Denoting the (Bohr) class of a.p. functions by $\mathfrak{u}$, we summarize these relations in

THEOREM 16.1. If $G=R_{1}, \mathfrak{W} \subset W^{p}$ and $\mathfrak{W} \cap S^{p}=\mathfrak{U}$ for every $p \geqq 1$.

\section{BIBLIOGRA PHY}

Additional references are given in [33].

1. L. Alaoglu and G. Birkhoff, General ergodic theorems. Ann. of Math. (2) vol. 41 (1940) pp. 293-309.

2. S. Banach, Theorie des opérations linéaires, Warsaw, 1932.

3. A. S. Besicovitch, Almost periodic functions, Cambridge, England, 1932.

4. S. Bochner, Vorlesungen über Fouriersche Integrale, Leipzig, 1932.

5. H. Bohr and E. F $\phi$ lner, On some types of functional spaces, Acta Math. vol. 76 (1945) pp. 31-155.

6. D. G. Bourgin, Linear topological spaces, Amer. J. Math. vol. 65 (1943) pp. 637-659.

7. J. W. Calkin, Abstract symmetric boundary conditions, Trans. Amer. Math. Soc. vol. 45 (1939) pp. 369-442.

8. M. M. Day, Operations in Banach spaces, Trans. Amer. Math. Soc. vol. 51 (1942) pp. 583-608.

9. - Ergodic theorems for Abelian semi-groups, Trans. Amer. Math. Soc. vol. 51 (1942) pp. 399-412. 
10. (a) N. Dunford, Spectral theory. I. Convergence to projections, Trans. Amer. Math. Soc. vol. 54 (1943) pp. 185-217.

(b) - Spectral theory, Bull. Amer. Math. Soc. vol. 49 (1943) pp. 637-651.

11. N. Dunford and D. S. Miller, On the ergcdic theorem, Trans. Amer. Math. Soc. vol. 60 (1946) pp. 538-549.

12. N. Dunford and B. J. Pettis, Linear operations on summable functions, Trans. Amer. Math. Soc. vol. 47 (1940) pp. 323-392.

13. W. F. Eberlein, Weak compactness in Banach spaces I, Proc. Nat. Acad. Sci. U.S.A. vol. 33 (1947) pp. 51-53.

14. ——, Abstract ergodic theorems, Proc. Nat. Acad. Sci. U.S.A. vol. 34 (1948) pp. 4347.

15. M. Eidelheit, On isomorphisms of rings of linear operators, Studia Math. vol. 9 (1940) pp. 97-105.

16. I. Gelfand and M. Neumark, On the imbedding of normed rings into the ring of operators in Hilbert space, Rec. Math. (Math. Sbornik) N.S. vol. 12 (1943) pp. 197-213.

17. R. Godement, Les fonctions de type positif et la théorie des groupes, Trans. Amer. Math. Soc. vol. 63 (1948) pp. 1-84.

18. W. H. Gottschalk, Almost periodicity, equi-continuity, and total boundedness, Bull. Amer. Math. Soc. vol. 52 (1946) pp. 633-636. 246-269.

19. E. Hille, Remarks on ergodic theorems, Trans. Amer. Math. Soc. vol. 57 (1945) pp.

20. D. H. Hyers, Linear topological spaces, Bull. Amer. Math. Soc. vol. 51 (1945) pp. 1-21.

21. S. Kakutani, Two fixed-point theorems concerning bicompact convex sets, Proc. Imp. Acad. Tokyo vol. 14 (1939) pp. 242-245.

22. - Concrete representation of abstract (M)-spaces, Ann. of Math. (2) vol. 42 (1941) pp. 994-1024.

23. Y. Kawada, Über den Mittelwert der messbaren fastperiodischen Funktionen auf einer Gruppe, Proc. Imp. Acad. Tokyo vol. 19 (1943) pp. 264-266.

24. W. Maak, Eine neue Definition der fastperiodischen Funktionen, Abh. Math. Sem. Hamburgischen Univ. vol. 11 (1936) pp. 240-244.

25. J. von Neumann, Almost periodic functions in a group. I, Trans. Amer. Math. Soc. vol. 36 (1934) pp. 445-492.

26. R. S. Phillips, On weakly compact subsets of a Banach space, Amer. J. Math. 65 (1943) pp. 108-136.

27. D. A. Raikov, Harmonic analysis on commutative groups with the Haar measure and the theory of characters (Russian), Travaux de l'Institut Mathématique Stekloff vol. 14 (1945).

28. F. Riesz, Some mean ergodic theorems, J. London Math. Soc. vol. 13 (1938) pp. 274-278.

29. V. Smulian, On some problems of the functional analysis, C. R. (Doklady) Acad. Sci. URSS N.S. vol. 38 (1943) pp. 157-159.

30. B. de Sz. Nagy, On uniformly bounded linear transformations in Hilbert space, Acta Univ. Szeged. vol. 11 (1947) pp. 152-157.

31. J. W. Tukey, Convergence and uniformity in topology, Princeton, 1940.

32. A. Weil, L'integration dans les groupes topologiques et ses applications, Actualités Scientifiques et Industrielles, no. 869, Paris, 1940.

33. K. Yosida and S. Kakutani, Operator-theoretical treatment of Markoff's process and mean ergodic theorem, Ann. of Math. (2) vol. 42 (1941) pp. 188-228.

34. A. Zygmund, Trigonometrical series, Warsaw, 1935.

Institute For Advanced Study,

Princeton, N. J. 